\title{
Interactions between patients' experiences in mental health treatment and lay social network attitudes toward doctors in recovery from mental illness
}

\author{
BREA L. PERRY \\ Indiana University Department of Sociology, Indiana University Bloomington, Bloomington, Indiana, USA \\ and \\ Indiana University Network Science Institute, Indiana University Bloomington, Bloomington, Indiana, USA \\ (e-mail: blperry@indiana.edu) \\ ERIN PULLEN \\ Indiana University Network Science Institute, Indiana University Bloomington, Bloomington, Indiana, USA \\ (e-mail: elpullen@indiana.edu)
}

BERNICE A. PESCOSOLIDO

Indiana University Department of Sociology, Indiana University Bloomington, Bloomington, Indiana, USA and

Indiana University Network Science Institute, Indiana University Bloomington, Bloomington, Indiana, USA

(e-mail: pescosol@indiana.edu)

\begin{abstract}
Research in the area of social networks and health has demonstrated that lay social network members play a critical role in the early stages of the illness career, influencing key decisions and pathways to formal care. Here, we revisit and extend this body of work, examining how the lay social network context can moderate the influence of treatment experiences on recovery outcomes as the illness career unfolds. To achieve this goal, we address two research questions, drawing on a longitudinal sample of people making their initial contact with the mental health treatment system: First, we explore how treatment experiences, lay social network characteristics, and recovery outcomes change over 2 years, beginning with the point of entry into treatment. Second, we examine whether the relationship between perceived treatment experiences and recovery outcomes is contingent on characteristics of the lay network context in which clients are socially embedded, focusing on the network's cultural orientation toward medical professionals. We find that positive treatment interactions facilitate improved self-esteem, mastery, role functioning, recovery optimism, and global functioning when the lay network culture is pro-medical, but largely have null effects on the recovery process when the lay network is more hostile to medical professionals.
\end{abstract}

Keywords: sociology, mental health, egocentric networks, recovery, network culture, health, network dynamics, longitudinal methods

Nearly, 10 million adults in the United States live with serious mental illness, and estimates suggest that at least $40 \%$ do not receive adequate mental health treatment (NSDUH, 2014; 2009). The consequences of not receiving treatment are both dire and largely preventable - including greater illness severity, diminished prospects of long-term recovery, victimization, incarceration, and suicide. Empirical 
work consistently suggests that both lay and professional supporters play a critical role in health services utilization and recovery from an episode of mental illness. On the one hand, sociologists have established the importance of friends, family members, and other informal caregivers in shaping an individual's illness career, focusing largely on the resources flowing through networks (Carpentier \& Bernard, 2011; Perry \& Pescosolido, 2015; Pescosolido, 2006; Pescosolido et al., 1998a). On the other hand, a large literature in psychology has examined the influence of relationships with mental health treatment providers and experiences in treatment on outcomes (Adams et al., 2007; Cruz \& Pincus, 2002). However, these two lines of research have seldom been integrated to examine both formal and informal network contexts, in tandem or in interaction.

Here, we revisit and extend these two bodies of research, examining how the lay social network context can moderate the influence of treatment experiences on recovery outcomes as the illness career unfolds. To achieve this goal, we address two research questions, drawing on a longitudinal sample of egos (i.e. focal respondents) making their initial contact with the mental health treatment system: First, we explore how treatment experiences and attitudes, basic lay social network characteristics, and recovery outcomes change over time in the sample, beginning with the point of entry into treatment and ending about 2 years later. Consistent with contemporary views on recovery, we emphasize development of a positive self-concept, return to former social roles and identities, optimism about one's ability to manage serious mental illness in the future, and psychological, social, and occupational functioning (Markowitz, 2001). Second, we examine whether the relationship between perceived treatment experiences and recovery outcomes is contingent on characteristics of the broader lay network context in which providers and mental health clients are socially embedded. In conceptualizing the informal social safety net, we focus on the network's cultural orientation toward medical professionals.

\section{Background}

Formal and informal social networks are critical for individuals experiencing the onset, response to, and prolonged management of serious health problems (Abbott et al., 2012; Lively \& Smith, 2011). An extensive area of scholarship has focused on access to social resources through network ties, including the ways that differing forms of support can directly and indirectly shape mental and physical health outcomes. Emotional support from personal networks, for example, can engender a sense of belonging and positive feelings of self-esteem and mastery-important psychological mechanisms linking networks to health (Thoits, 2011; Uchino, 2004; Pernice-Duca, 2010; Bracke et al., 2008). Similarly, instrumental support (e.g. providing transportation or childcare) and informational support (e.g. advice about how to find the best medical care) can have an important impact on health behaviors, influencing health services utilization, treatment retention, and treatment compliance in the context of various health problems, including serious mental illness (Dracup, 2013; Perry \& Pescosolido, 2012; Pullen et al., 2014). Importantly, social resources can also buffer the effects of stressful life circumstances, enabling vulnerable individuals 
with otherwise limited resources to make choices that improve their health and wellbeing (Thoits, 2011).

Another way social relationships may influence individuals' health is through social regulation. Members of social networks may employ overt and subtle tactics to surveil, persuade, cajole, and regulate behaviors in routine circumstances and during the course of an unfolding health crisis. Research consistently finds that within marital relationships, for example, men are subject to substantial efforts by their partners to influence and control their health (Umberson, 1992). This has been shown to have salubrious effects, such as reductions in unhealthy behaviors like substance use and adoption of positive health behaviors like proper diet and exercise (Lewis \& Butterfield, 2007; Duncan et al., 2006). However, evidence also suggests that social regulation efforts may be perceived as unnecessary, bothersome, or coercive, having neutral or even negative effects on treatment seeking and other health outcomes in some contexts (Franks et al., 2006; Pescosolido et al., 1998a; Wild et al., 2006; Lewis, 2005). In fact, individuals may exercise agency in how they consciously and subconsciously draw on network ties and resources, "pushing back" against networks' regulatory efforts and selectively activating relationships that are perceived more favorably or that offer desired benefits (Perry \& Pescosolido, 2012; Perry \& Pescosolido, 2015; Hurlbert et al., 2000).

Social networks are also instrumental in influencing individuals' orientations toward health and health care - a function often conceptualized as access to cultural health capital. Shim (2010) defines cultural health capital as "the repertoire of cultural skills, verbal and nonverbal competencies, attitudes and behaviors, and interactional styles" (p. 1) that influence relationships between providers and patients, as well as outcomes of health services. The particular set of preferences, beliefs, and attitudes about health and illness, help-seeking, and formal health care that a person holds structures behaviors around health and health care utilization (Bourdieu, 1977; 1990; Lo \& Stacey, 2008). In particular, cultural health capital patterns conscious and unconscious beliefs about what behavior constitutes a possible and preferable response to a health crisis (Perry \& Pescosolido, 2015). Importantly, individuals' orientations toward health and health care are developed through interaction with social network ties and exposure to the cultural capital made available by these ties (Lo \& Stacey, 2008). Consequently, individual agency and behavior operates within the boundaries of the cultural health capital accessible through personal social networks and relationships, both past and present (Perry \& Pescosolido, 2015).

\subsection{Social networks and recovery from serious mental illness}

Before discussing the role of social networks in recovery, it is useful to briefly outline how mental health professionals and consumers currently conceptualize recovery from serious mental illness. The psychiatric consumer/survivor movement was instrumental in reforming modern notions of recovery (Davidson et al., 2005). Previously, recovery had been defined as a reduction in symptoms of mental illness to the extent that they no longer interfere with daily functioning. However, research indicating that only about one-quarter to two-thirds of individuals with serious mental illness ever achieve this benchmark led to new ways of thinking about mental illness recovery. Specifically, the concept of recovery was shifted toward a 
process of overcoming “... poverty, substandard housing, isolation, unemployment, loss of valued social roles and identity, loss of sense of self and purpose in life ... in order to retain or resume some degree of control over their own lives (481: Davidson et al., 2005)." In short, recovery does not mean freedom from symptoms of mental illness, but a reduction in the adverse consequences of symptoms (and treatment), including investment in a life with value and meaning (Hendryx et al., 2009; Mezzina et al., 2006; Padgett et al., 2008).

Evidence suggests that social network ties may have a strong and lasting influence on the entire recovery process, from initial interpretations of symptoms to compliance with mental health professionals' advice. The onset of serious mental illness precipitates engagement with lay social networks, which shape key decisions about how to respond to acute symptoms and crisis (Pescosolido, 1992). In early work on pathways into mental health services, Kadushin (1958) noted that a meaningful proportion of people in treatment are influenced by friends or family members to seek care. He argued that accepting one's problems as psychiatric in nature requires a redefinition of the self as a person with mental illness that is heavily dependent on the appraisals of significant others (1955; Kadushin, 1966). Mirroring modern notions of cultural health capital, Kadushin's research demonstrated that there were "friends and supporters of psychotherapy" - a personal network characterized by a set of shared norms and values, which, as a whole, constituted a favorable orientation toward psychotherapy. Members of these networks held less stigmatizing attitudes toward emotional problems and psychiatric services, were more apt to respond to such problems with sympathy and understanding, and were more likely to discuss their own mental health symptoms and treatment with others. Not surprisingly, then, Kadushin found that membership in this kind of network shaped respondents' definitions of psychiatric problems, pathways to care, and persistence in psychotherapy.

Contemporary research supports Kadushin's findings, demonstrating the critical role of lay caregivers in promoting or obstructing entry into mental health services (Carpentier et al., 1999; Cusack et al., 2004; Hansen \& Aranda, 2012; Lindsey et al., 2010; Pescosolido et al., 1998a; Pescosolido et al., 1998b; Spoont et al., 2014; Thoits, 2011; Villatoro \& Aneshensel, 2014). In addition, lay supporters who advocate formal mental health services as the appropriate solution to symptoms of mental illness may be important sources of validation of entry into care and support for the treatment process. Consequently, the attitudes and values to which patients are exposed may also influence the outcomes of treatment (Padgett et al., 2008). Social networks hold their own cultural disposition toward the medical system and health professionals, and this can have an important bearing on how, when, and under what circumstances individuals engage with the treatment system, and the relative impact this engagement has on their response to illness.

A central argument of this paper is that the strength of the therapeutic relationship or the efficacy of mental health services may be compromised in the context of a lay social network that has a negative orientation toward psychiatry or medical professionals. Since cultural health capital informs sense-making during interactions with formal treatment providers (Lo \& Stacey, 2008), exposure to a shared culture of mistrust and skepticism toward formal health care providers in the individual's community network may reduce engagement, motivation, and 
compliance in treatment. Further, acting contrary to a shared health cultureeven if that action is putatively health promoting (e.g. formal help-seeking) - may prompt sanction from informal network ties and threaten established, supportive relationships (Bourdieu, 1986; Centola, 2010; Trotter et al., 1995). Moreover, holding beliefs that are dissimilar to close members of one's network can create cognitive imbalance that provokes stress, initiating conscious or unconscious actions that reduce conflict (Heider, 1958; Hummon \& Doreian, 2003; Lazer et al., 2010). These actions may include severing relationships, changing one's own views to more closely align with others', or working to persuade others to change their beliefs. Because people with mental illness have high support needs, they may be particularly motivated to model their orientation toward formal health care on that of their social networks to maintain cognitive balance and reinforce these supportive relationships (Kenny, 1994; Lazer et al., 2010). In other words, in addition to influencing pathways into treatment, one's lay social network may also affect the efficacy of mental health services, facilitating authentic engagement with professionals and the therapeutic process, and increasing adherence to treatment plans and goals.

There is some existing research that supports this central hypothesis, suggesting that the people mental health patients turn to in crisis can have significant implications for recovery (Padgett et al., 2008). Turning to weaker network ties or those that are unsupportive of formal treatment, for example, has been shown to correspond with lower social satisfaction, quality of life, and fulfillment of social roles, all of which represent important dimensions of recovery from serious mental illness (Perry \& Pescosolido, 2015). Conversely, embeddedness in a network that is supportive of formal medical treatment corresponds to more promising recovery outcomes, including improved mental health functioning (Perry \& Pescosolido, 2015). Along the same lines, having networks that promote access to accurate, evidencebased information about managing illness symptoms can have beneficial outcomes (Cummings \& Kropf, 2009).

\subsection{The role of mental health treatment in recovery}

As individuals manage an episode of mental illness, interactions with health care professionals, the quality of these relationships, and the trust they place in experts may have an important bearing on their behavior and attitudes toward treatment (Horvath et al., 2011; Laugharne \& Priebe, 2006). About one in three individuals with serious mental illness who have contact with mental health services withdraw from care (Kreyenbuhl et al., 2009). Because continued contact with treatment providers is associated with positive health outcomes among this population, efforts to understand characteristics that correspond to treatment dropout, retention, and continuity of care is critical. A large literature focuses on the therapeutic alliance and satisfaction with providers (Rogers, 1951; Cruz \& Pincus, 2002; McCabe \& Priebe, 2004). Findings demonstrate that ongoing positive interaction between patients and their providers corresponds to greater compliance with prescribed medications and continuity of care (Curtis et al., 2010; Adair et al., 2005). Moreover, a positive relationship between mental health providers and their patients in community mental health clinics predicts greater patient readiness to take on the role of managing their wellbeing and treatment (Allen et al., 2015). In all, satisfaction with 
the treatment patients receive is associated with a reduction in symptom burden (Adams et al., 2007), perhaps reflecting the efficacy of care. Alternatively, in the presence of a weak therapeutic bond and dissatisfaction with mental health services, prescribed treatments may be perceived as coercive, contributing to non-compliance, disengagement from treatment, and, ultimately, poorer recovery outcomes (Roe et al., 2009; Johansson \& Eklund, 2006).

Patients' opinions about medical professionals and psychiatric treatment may also influence their perceptions of its efficacy. Research indicates that negative attitudes among patients toward treatment correspond to a weaker therapeutic alliance (Barrowclough et al., 2010), whereas a strong therapeutic alliance is built on a foundation of trust in mental health providers and the treatment process. Because individuals' attitudes toward mental illness and treatment are formed and evolve through interaction with family, friends, and other network members (Pescosolido, 1992), it is likely that the culture of the lay social network shapes the treatment process (Perry et al., 2016). Whether the lay network and the treatment network are in sync or at odds may be critical for continuity of care and adherence with medical regimens (Pescosolido, 1996). However, little is known about how the broader lay social context influences relationships between treatment providers and their patients, and most importantly, how network interactions shape health and social outcomes for individuals.

In the current study, we leverage egocentric social network methods to examine how lay social networks influence the outcomes of treatment episodes. We advance the notion that lay and treatment environments may operate in harmony or work at cross purposes. In addition, we extend existing research on lay social network effects in mental health to a later stage of the illness career, examining the outcomes of mental health treatment rather than patterns of utilization alone. This strategy provides a more rigorous and comprehensive test of the influence of network context, revealing whether the presence of lay supporters shapes recovery after entry into treatment. While the network perspective on the illness career has existed since Kadushin (1958), this is, to our knowledge, the first study to empirically examine whether the relationship between perceived treatment experiences and recovery outcomes over time is contingent on cultural attitudes toward medical professionals present in the social networks of mental health clients. In addition to having implications for network theory, this analysis may provide important insights about the role of family and friends in shaping outcomes of care by influencing the patients' views of treatment and consequences of following medical advice.

\section{Data and methods}

\subsection{Sample}

The Indianapolis Network Mental Health Study (INMHS) uses egocentric network methods to identify relationships between the early mental illness career and social network dynamics. Though fielded between 1990 and 1997, the INMHS is the only network study of first-time mental health patients, and remains one of the most detailed longitudinal, network-based studies of people with mental illness. However, because respondents were recruited following entry into treatment, our analyses 
cannot address the social network factors affecting who enters formal treatment and why. ${ }^{1}$ A large population study would be required to capture enough untreated individuals with serious mental illness to trace utilization trajectories, making it cost-prohibitive to conduct the kind of in-depth investigation of social networks undertaken in the INMHS. For this reason, the INMHS focused on "first-timers," attempting to capture people with serious mental illness as close to entry into the treatment career as possible.

Respondents for the INMHS were recruited from two large public and private hospitals (including an embedded community mental health center). Recruitment, including assessment for inclusion criteria, occurred in emergency rooms and crisis centers. Some respondents were subsequently hospitalized, but the majority were referred for outpatient treatment. The following inclusion criteria were used: Being aged 18 or older, receiving an Axis I psychiatric research diagnosis (based on the Structured Clinical Interview for DSM-III-R; Spitzer et al., 1990), experiencing first major contact with the mental health treatment system ("When was the first time you saw someone for emotional or psychiatric problems?"), and having a mental illness history of no longer than 2 years ("When did this begin, or when did you first notice something was wrong?"). All new patients meeting these criteria were asked to participate in face-to-face interviews, which were conducted within 3 months of initial contact. Those not meeting these screening criteria were not invited to participate (e.g. patients who had previously sought medical help for psychiatric problems). The response rate was $66.4 \%$.

A total of 173 individuals (i.e. egos, or focal persons) participated in the first round of face-to-face interviews. A total of 26 respondents $(15 \%)$ were dropped due to missing data on study variables, with 23 of these dropped due to missing data on household income. ${ }^{2}$ After dropping cases with missing data, the effective sample size is 147 respondents, who provided information about 2,080 members of their network (i.e. alters), including friends, family members, coworkers, neighbors, mental health treatment providers, and others. Each focal respondent was interviewed up to three times - shortly after the point of entry into treatment (baseline), approximately 10 months after baseline, and approximately 24 months after baseline. These data are unbalanced due to attrition from the study over time, though respondents were contacted at wave 3 even if they did not participate in wave 2. This strategy resulted in 273 person observations, such that each focal respondent was interviewed about two times, on average. Alters were identified and linked across waves of the study.

1 Although the networks of people with mental illness who did enter treatment likely look different from those who did not, we should note that INMHS respondents entered treatment in many different ways. Some came voluntarily, others were coerced by family members, and some were picked up by police and brought to the emergency department involuntarily (Pescosolido et al., 1998a).

2 We conducted a comparison of cases retained in the analysis and cases dropped due to missing data. This analysis suggests that respondents dropped due to missing data are disproportionately likely to be men $(p<0.01)$, white $(p<0.05)$, not currently married $(p<0.01)$, and to have smaller social networks $(p<0.001)$. However, there are no significant differences on key independent or dependent variables in this analysis: treatment satisfaction, network trust in doctors, or recovery outcomes. Moreover, because a fixed effects strategy is used to model within-person change over time, all time-invariant attributes (including gender and race) are controlled. 


\subsection{Measures}

\subsubsection{Dependent variables}

Five dependent variables are employed as indicators of overall functioning and wellbeing, consistent with modern definitions of recovery from serious mental illness. Four of these variables are standardized since the natural units are uninterpretable, while the natural units are retained for the fifth (Global Assessment of Functioning (GAF)). Rosenberg's self-esteem scale (Rosenberg, 1965) measures feelings of selfworth, and is comprised of five items measured on a five-point Likert scale (e.g. I am able to do things as well as most other people.). Non-missing items are averaged such that higher values signify better self-esteem $(\mathrm{alpha}=0.83)$. Improvements in self-esteem over time are associated with recovery, as feelings of worthlessness and lack of purpose associated with serious mental illness abate.

Mastery reflects the extent to which a person's life chances are perceived to be under their own control, as opposed to being left up to fate. This is measured using three items (e.g. What happens to me in the future mostly depends on me.) assessed on a five-point Likert scale, where non-missing items are averaged to produce a scale ranging from 1 to 5 (Pearlin \& Schooler, 1978). Higher values indicate stronger feelings of mastery. Mastery over one's environment should increase in conjunction with recovery as people with mental illness begin to gain a sense of control over their symptoms.

Perceived success at functioning in various social roles is also assessed using a scale that includes 25 items asking "how good or successful" the respondent is at being (social role). It includes family roles (e.g. parent, son/daughter, sibling), work and volunteer roles, friend, neighbor, and church member, etc. Responses range from "poor" to "good" (higher $=$ more success) and are averaged (alpha $=0.81$ ). A key feature of recovery is a successful return to previously held social roles, such as employee and friend.

A recovery optimism scale assesses respondents' feelings about how they see themselves in the future, and includes items like, "I will continue to be dependent on my family," and "I will be able to function very well in the community." Six items are measured on a five-point Likert scale and averaged (negative items reverse coded) such that higher values equal more optimism about their mental health recovery (alpha $=0.70$ ). As respondents recover from serious mental illness, we expect improvements in their perceptions of their ability to function independently in the future.

Finally, GAF is included as an indicator of how well a person adapts to challenges of daily living. It is a scale used by mental health professionals and researchers to rate psychological, social, and occupational functioning. With minimal training, interviewers are able to subjectively but reliably evaluate the functioning of respondents at the conclusion of the interview (Startup et al., 2002). It ranges from 0 to 100 , where higher values signify better functioning. We expect increasing GAF scores to correspond to recovery from mental illness.

\subsubsection{Independent variables}

As noted above, data were collected on respondents' alters across a broad range of social domains. Each domain had a corresponding name generator, with no limitations on the number or types of people respondents could name. The 13 name 
generators used to elicit network ties include: contacts during the illness episode (e.g. people that ego talked to, got help or advice from, etc.), household members, partner/spouse/boy or girlfriend, family members in regular contact, coworkers, classmates, fellow volunteers, close friends, casual friends, enemies, important matters discussants, health matters discussants (Perry \& Pescosolido, 2010), people with similar problems, significant hospital/clinic staff and other treatment providers, and people who help or hinder medication compliance. This approach provides a near inventory of people who have semi-regular contact with respondents (i.e., total network), including ties of great significance (e.g. core supporters) as well as those who may play a more peripheral or unidimensional role (e.g. neighbors, coworkers, etc.). For additional details on name generators and scales used in the INMHS, full versions of the instruments are available on the study website (http://www.indiana.edu/ icmhsr/instruments.html).

Measures of network characteristics are computed by aggregating information provided about each alter. All models control for social network size, which is the number of unique alters mentioned in response to any name generator in each wave. ${ }^{3}$ Mean network trust in physicians ${ }^{4}$ is based on an item asking how much each alter "trusts doctors to take care of people's problems." It is measured on a three-point Likert scale and averaged across all alters in a network, resulting in a potential range of 1-3. When a respondent selected "don't know" on this item for a particular alter, the alter-level observation is effectively coded to the mean for that ego network due to aggregation. Consequently, missing data at the alter level do not contribute explanatory power to the models, consistent with a lack of familiarity with an alter's attitudes toward doctors. ${ }^{5}$ Also, mental health professionals are eliminated prior to calculating mean network trust in physicians, consistent with our goal of measuring lay network attitudes. This strategy also avoids the potential confounding effects of number of mental health professionals in the network on recovery outcomes.

To determine the extent to which the lay network's trust in physicians moderates the influence of the treatment experience over and above one's own attitudes toward the medical system, we control for respondents' own confidence in physicians. This

3 Other network variables were initially included in models as controls, but were later removed due to non-significance and having no effect on the key interactions of interest. These include percent of the network composed of kin and percent composed of friends.

4 Accuracy of proxy reporting (i.e. ego reporting on the attitudes of alters) is always a concern in network research. To assess the possibility that findings are driven by inaccurate evaluations of weak ties (e.g. neighbors), we replicated our multivariate analyses using only core networks under the assumption that respondents will be more likely to be able to accurately report on these alters' attitudes toward doctors (Gant et al., 1999). These core networks included people they talk to about health matters, close friends, family members, and people they are "very close" to. Four out of five interactions reported in the paper are significant and similar in magnitude when only core network attitudes are used to predict recovery outcomes, supporting the validity of the trust in doctors measure. More broadly, it is important to note that in egocentric research designs, accuracy is not necessarily the goal. Explanations for social processes often hinge on ego imitating, adopting, or being influenced by what she perceives others' behaviors and attitudes to be. In short, ego's perceptions are the proximal cause, and accuracy may be irrelevant.

5 Respondents replied "don't know" for $18 \%$ of alters, on average. In addition, $67 \%$ of respondents report not knowing whether at least one network member has trust in physicians. We attempted to model the effects of "don't know" responses to assess whether uncertainty about network attitudes toward doctors influences recovery outcomes or moderates treatment satisfaction. Very few results were significant and there were no clear patterns, but this is an interesting and potentially important topic for future research. 
variable is computed by taking the average of five items that scale together in exploratory factor analysis, including "I often doubt some of the things doctors say they can do" and "for most kinds of illness, it is the doctor who can help you the most." This confidence in physicians scale ranges from 1-5 and higher values indicate more confidence (alpha $=0.65$ ).

We conduct growth models of recovery where an important component of the analysis is the effect of time. Time is indexed using actual months in the treatment career, measured as time since baseline recruitment and first entry into treatment. Although efforts were made to interview respondents at 10-month and 24-month follow-ups, some interviews were conducted earlier or later due to the difficulty of scheduling or locating respondents. Using a measure of real time rather than study wave provides a more accurate measure of progression through the treatment career. Months in treatment is coded to years in treatment (to two decimal places) to improve the interpretation of coefficients.

Time-variant sociodemographic characteristics of respondents (e.g. educational attainment, income, employment status, and marital status) can shape the structure and function of personal community networks over time, as well as recovery from mental illness (Ajrouch et al., 2001; Marsden, 1987; Peek \& O’Neill, 2001). Consequently, we consider these variables as controls in regression analyses. While time-invariant sociodemographic characteristics (e.g. gender, race, diagnosis) are considered for the purposes of providing sample descriptive statistics, they are not included in multivariate regression models due to the fixed effects modeling strategy described below. Time-variant socioeconomic status is measured using years of education and categorical household income coded to the midpoint at each wave of the study. Marital status is measured using a binary variable representing currently married, and employment with a binary variable reflecting full-time employment. Cell sizes are insufficient for more fine-grained analysis of marital and employment status. All models also control for a binary indicator of current mental health treatment, which is 1 if the ego respondent is currently receiving mental health services and 0 otherwise. All respondents were receiving treatment at baseline since they were recruited from treatment facilities, though not all were in treatment at the time of follow-up interviews.

Treatment experiences are measured at the ego level using a single scaled variable. It assesses satisfaction with various aspects of treatment, including overall assistance received at the treatment facility (hospital or $\mathrm{CMHC}$ ), help linking to community services, help coping with crisis, amount of contact with treatment providers, and support and comfort offered by treatment providers. All items are measured on a five-category Likert scale. All valid responses are averaged to compute a scale with a potential range of 1 to 5 , where higher values signify more satisfaction with various aspects of treatment (alpha $=0.95$ ). We expect more positive treatment experiences to be associated with better recovery outcomes.

\subsection{Analysis}

Two-level fixed effects models are used with Level-1 observations over time nested in Level-2 ego respondents. These models adjust for the lack of independence between observations nested within egos and alters, and are appropriate for unbalanced 
and longitudinal data. A major advantage of fixed effects models is that they address omitted variable bias, which is an important threat to causal arguments in social influence studies (Allison, 2005). They are often called "within-person" models because all between-person variation is conditioned out of the regression equation, and each person effectively serves as their own control in a model of intra-individual change over time. Consequently, fixed effects models control for all observed and unobserved heterogeneity in time-invariant (i.e. stable) characteristics, including anything that happened in the past (e.g. what networks looked like prior to entering treatment) and variables like genetic risk for psychiatric disorders. However, a disadvantage of fixed-effects models is that they sacrifice efficiency for unbiased parameter estimates, making it more difficult to detect significant results compared to random effects models (Clark \& Linzer, 2015). Consequently, variables like marital status that are highly predictive of mental health outcomes in betweenperson analyses may not be statistically significant in a fixed effects model either because (1) they don't change much over time within persons; or (2) all stable and past characteristics that influence both likelihood of entry into marriage and wellbeing are controlled.

The analysis proceeds in two steps. First, to assess change over time in key variables in this analysis, we conduct multilevel regression analyses predicting treatment variables, network characteristics, and recovery outcomes using the time variable (i.e. years in the treatment career), controlling for time-variant sociodemographic variables. We present predicted values at baseline, 1 year, and 2 years after entry into treatment to indicate how each variable changes over time, as well as significance tests for the time variable.

Second, we employ fixed effects regression models to examine how treatment experiences and network trust in the institution of medicine is associated with recovery outcomes, beginning with the point of entry into mental health treatment and ending about 2 years later. We present both main effects and interaction models. Interactions are detected using pooled regression models with an interaction term. The network trust variable (the moderator) is divided into tertiles for the purposes of testing the interaction since both variables being interacted are continuous. The significance of interactions is determined using Chow-type tests of the equality of coefficients. To facilitate interpretation of the interaction models, predicted values on standardized dependent variables are presented across treatment experiences and over values of mean network trust in doctors.

All models control for time-variant sociodemographic variables that could confound the relationship between attitudes toward treatment and recovery outcomes. We also include a binary variable indicating whether the respondent is currently in treatment. This ensures that being in any treatment per se is not confounded with evaluations of treatment. We also model the effects of aggregated mean network trust in doctors, excluding mental health treatment providers. We use mean trust in physicians because, of the measures available, this variable most closely operationalizes cultural attitudes that are consistent with network confidence in the institution of medicine. All models control for network size because smaller networks tend to be more homogenous with respect to attitudes. Models also control for respondent confidence in physicians to avoid the confounding effects of one's own attitudes, which may influence reporting of alters' attitudes, and to isolate network effects. 
Table 1. Sample descriptive statistics at baseline, INMHS $(n=147)$.

\begin{tabular}{lcrrc}
\hline \hline & Proportion & Mean & SD & Range \\
\hline $\begin{array}{l}\text { Respondent characteristics } \\
\text { Female }\end{array}$ & 0.68 & & & \\
White & 0.76 & & & \\
Education (years) & & 11.64 & 1.97 & $6.00-16.00$ \\
Household income (\$1K) & & 24.19 & 19.60 & $2.50-65.00$ \\
Works fulltime & 0.49 & & & \\
Currently married & 0.33 & & & \\
Diagnosis & & & & \\
Bipolar & 0.08 & & & \\
Schizophrenia & 0.09 & & & \\
Major depression & 0.55 & & & \\
Other & 0.28 & & & \\
Confidence in doctors & & 3.52 & 0.50 & $2.20-5.80$ \\
Receiving treatment & 1.00 & & & \\
Treatment satisfaction & & 3.65 & 0.87 & $1.00-5.00$ \\
Social network characteristics & & & & \\
Network size & & 20.35 & 9.41 & $7.00-58.00$ \\
Mean trust in doctors & & 2.24 & 0.41 & $1.00-3.00$ \\
Recovery outcomes & & & \\
Self-esteem & & 3.37 & 0.81 & $1.00-5.00$ \\
Mastery & & 3.20 & 0.69 & $1.33-5.00$ \\
Role functioning & & 3.73 & 0.65 & $1.00-5.00$ \\
Recovery optimism & 3.56 & 0.50 & $2.20-5.00$ \\
Global Assessment of Functioning & & 47.08 & 14.56 & $10.00-78.00$ \\
\hline \hline
\end{tabular}

The scaled dependent variables are relatively normally distributed, making linear fixed effects regression an appropriate estimation procedure. These scales are standardized to facilitate interpretation, except for GAF, which is measured in interpretable units. Variance inflation factors and Cook-Weisberg tests indicate that levels of multicollinearity and heteroskedasticity are unproblematic.

\section{Results}

Table 1 presents sample descriptive statistics at baseline. These indicate that $68 \%$ of respondents in the analysis sample are women; $76 \%$ are White and $24 \%$ are Black. Respondents range in age from 18 to 72 (mean $=30.9$ years) with an average education of 11.6 years and household income of $\$ 24,190$. About one-third are currently married. About $8 \%$ of respondents are diagnosed with bipolar disorder, $55 \%$ with major depression, $9 \%$ with schizophrenia, schizoaffective disorder or similar, and $28 \%$ with less severe disorders. Though these data are not shown in Table 1, most respondents lived and worked independently (e.g. 86\% lived alone, with a spouse/partner, or with a roommate; $78 \%$ were currently working, in school full time, or keeping house).

Table 2 presents predict values for key independent and dependent variables over time, controlling for time-variant sociodemographic characteristics of respondents. Respondents' confidence in doctors is predicted to be slightly better than neutral at the point of entry into treatment (3.56 on a five-point scale), and the predicted 
Table 2. Change over time in predicted values ${ }^{\dagger}$ for treatment, network, and recovery variables, INMHS $(n=147)$.

\begin{tabular}{lcccr}
\hline \hline & Baseline & 12 months & 24 months & Sig. \\
\hline Confidence in doctors & $3.56(3.48-3.64)$ & $3.49(3.44-3.53)$ & $3.41(3.30-3.52)$ & NS \\
Treatment satisfaction & $3.62(3.48-3.76)$ & $3.61(3.53-3.69)$ & $3.59(3.40-3.78)$ & NS \\
Network size & $22.07(21.81-22.33)$ & $22.64(22.49-22.79)$ & $23.21(22.86-23.56)$ & $* * *$ \\
Network trust in docs & $2.23(2.14-2.32)$ & $2.20(2.14-2.25)$ & $2.17(2.03-2.30)$ & NS \\
Self-esteem & $3.46(3.36-3.57)$ & $3.60(3.54-3.67)$ & $3.75(3.60-3.90)$ & $*$ \\
Mastery & $3.22(3.12-3.33)$ & $3.42(3.35-3.48)$ & $3.61(3.46-3.76)$ & $* * *$ \\
Role functioning & $3.76(3.67-3.84)$ & $3.81(3.76-3.86)$ & $3.86(3.75-3.97)$ & NS \\
Recovery optimism & $3.58(3.51-3.66)$ & $3.55(3.51-3.60)$ & $3.52(3.42-3.62)$ & NS \\
GAF & $50.86(48.36-53.36)$ & $61.49(60.04-62.93)$ & $72.12(68.67-75.56)$ & $* * *$ \\
\hline \hline
\end{tabular}

${ }^{\dagger}$ Predicted values are derived from fixed effects regression models controlling for timevariant respondent characteristics in Table 1. Significance based on $p$-value of coefficient for time. Confidence intervals are shown in parentheses.

value for the sample declines slightly (but not significantly) over time. Treatment satisfaction in the sample is also fairly positive, with a predicted value of 3.62 on a five-point scale, and is stable over time. The predicted network size for the sample increases significantly over time from about 22 alters at baseline to over 23 after 2 years in the treatment career $(p<0.001)$. This may be indicative of a reduction in social withdrawal that is characteristic of major depression (the diagnosis held by over $50 \%$ of the sample). Network mean trust in physicians is predicted to be slightly higher than the scale midpoint (2.24 on a three-point scale), indicating that most network members have a neutral or positive orientation toward medical professionals at baseline, and the predicted value changes very little over time for the sample as a whole.

With respect to recovery outcomes, changes in the predicted values for the sample over time are indicative of improvements in mental health. The predicted value of self-esteem increases significantly over time from 3.46 at baseline to 3.75 after 2 years (five-point scale; $p<0.05$ ). Perceptions of mastery also increase significantly from a predicted value of 3.22 on a five-point scale at entry into treatment to 3.61, 2 years later $(p<0.001)$. Predicted role functioning increases slightly, but not significantly, while recovery optimism declines slightly (not significant). Finally, and most remarkably, the predicted value for the GAF scale, which has a potential range from 1 to 100 , increases from 50.86 at the point of entry into treatment to $72.12,2$ years later $(p<0.001)$.

\subsection{Effects of treatment experiences and network characteristics on recovery outcomes}

Regression of five indicators of mental illness recovery on treatment experiences and social network trust in doctors is presented in Tables 3 and 4. As shown in Models 1 and 3 of Table 3, increasing confidence in doctors is associated with lower levels of self-esteem $(b=-0.39, p<0.05)$, while better experiences in treatment are associated with higher levels of mastery $(b=0.31, p<0.01)$. Additionally, Model 3 
Table 3. Fixed effects linear regression ${ }^{\dagger}$ of self-esteem, mastery, and role functioning on the interaction of treatment satisfaction and social network trust in doctors, INMHS.

\begin{tabular}{|c|c|c|c|c|c|c|}
\hline & \multicolumn{2}{|c|}{ Self-esteem } & \multicolumn{2}{|c|}{ Mastery } & \multicolumn{2}{|c|}{ Role functioning } \\
\hline & 1 & 2 & 3 & 4 & 5 & 6 \\
\hline & $\mathrm{B}(\mathrm{SE})$ & $\mathrm{B}(\mathrm{SE})$ & $\mathrm{B}(\mathrm{SE})$ & $\mathrm{B}(\mathrm{SE})$ & $\mathrm{B}(\mathrm{SE})$ & $\mathrm{B}(\mathrm{SE})$ \\
\hline Time (years) & $\begin{array}{c}0.016 \\
(0.091)\end{array}$ & $\begin{array}{c}0.030 \\
(0.089)\end{array}$ & $\begin{array}{c}0.033 \\
(0.099)\end{array}$ & $\begin{array}{c}0.046 \\
(0.097)\end{array}$ & $\begin{array}{c}-0.065 \\
(0.083)\end{array}$ & $\begin{array}{c}-0.059 \\
(0.082)\end{array}$ \\
\hline \multicolumn{7}{|l|}{ Ego characteristics } \\
\hline Education (years) & $\begin{array}{c}0.253 \\
(0.241)\end{array}$ & $\begin{array}{c}0.383 \\
(0.240)\end{array}$ & $\begin{array}{c}0.045 \\
(0.261)\end{array}$ & $\begin{array}{c}0.170 \\
(0.262)\end{array}$ & $\begin{array}{c}0.086 \\
(0.219)\end{array}$ & $\begin{array}{c}0.155 \\
(0.220)\end{array}$ \\
\hline Household income ( $\$ 10 \mathrm{~K})$ & $\begin{array}{c}0.015 \\
(0.060)\end{array}$ & $\begin{array}{c}0.004 \\
(0.059)\end{array}$ & $\begin{array}{c}0.108 \\
(0.065)\end{array}$ & $\begin{array}{c}0.098 \\
(0.064)\end{array}$ & $\begin{array}{c}-0.005 \\
(0.054)\end{array}$ & $\begin{array}{r}-0.010 \\
(0.054)\end{array}$ \\
\hline Working fulltime & $\begin{array}{c}0.074 \\
(0.165)\end{array}$ & $\begin{array}{c}0.0362 \\
(0.162)\end{array}$ & $\begin{array}{c}-0.111 \\
(0.179)\end{array}$ & $\begin{array}{r}-0.146 \\
(0.177)\end{array}$ & $\begin{array}{c}-0.135 \\
(0.150)\end{array}$ & $\begin{array}{r}-0.145 \\
(0.149)\end{array}$ \\
\hline Currently married & $\begin{array}{c}0.065 \\
(0.291)\end{array}$ & $\begin{array}{c}0.159 \\
(0.287)\end{array}$ & $\begin{array}{c}-0.518 \\
(0.316)\end{array}$ & $\begin{array}{c}-0.421 \\
(0.314)\end{array}$ & $\begin{array}{c}0.493 \\
(0.265)\end{array}$ & $\begin{array}{r}0.576 * \\
(0.264)\end{array}$ \\
\hline Confidence in doctors & $\begin{array}{c}-0.386 * \\
(0.164)\end{array}$ & $\begin{array}{c}-0.408 * \\
(0.161)\end{array}$ & $\begin{array}{c}-0.142 \\
(0.178)\end{array}$ & $\begin{array}{c}-0.164 \\
(0.176)\end{array}$ & $\begin{array}{c}-0.236 \\
(0.151)\end{array}$ & $\begin{array}{r}-0.256 \\
(0.149)\end{array}$ \\
\hline Receiving treatment & $\begin{array}{c}0.092 \\
(0.160)\end{array}$ & $\begin{array}{c}0.135 \\
(0.158)\end{array}$ & $\begin{array}{c}0.031 \\
(0.174)\end{array}$ & $\begin{array}{c}0.069 \\
(0.173)\end{array}$ & $\begin{array}{c}0.048 \\
(0.146)\end{array}$ & $\begin{array}{c}0.056 \\
(0.146)\end{array}$ \\
\hline Treatment satisfaction & $\begin{array}{c}0.138 \\
(0.096)\end{array}$ & $\begin{array}{c}-0.062 \\
(0.119)\end{array}$ & $\begin{array}{l}0.313 * * \\
(0.104)\end{array}$ & $\begin{array}{c}0.118 \\
(0.129)\end{array}$ & $\begin{array}{c}0.077 \\
(0.087)\end{array}$ & $\begin{array}{c}-0.042 \\
(0.109)\end{array}$ \\
\hline Netw & & & & & & \\
\hline Network size & $\begin{array}{c}-0.030 \\
(0.051)\end{array}$ & $\begin{array}{c}-0.025 \\
(0.050)\end{array}$ & $\begin{array}{c}-0.023 \\
(0.055)\end{array}$ & $\begin{array}{c}-0.019 \\
(0.055)\end{array}$ & $\begin{array}{c}0.041 \\
(0.047)\end{array}$ & $\begin{array}{c}0.040 \\
(0.046)\end{array}$ \\
\hline Mod. trust in doctors $\ddagger$ & $\begin{array}{r}-0.150 \\
(0.151)\end{array}$ & $\begin{array}{c}-0.912 \\
(0.493)\end{array}$ & $\begin{array}{r}-0.057 \\
(0.164)\end{array}$ & $\begin{array}{c}-0.702 \\
(0.538)\end{array}$ & $\begin{array}{c}0.081 \\
(0.137)\end{array}$ & $\begin{array}{c}0.107 \\
(0.452)\end{array}$ \\
\hline High trust in doctors ${ }^{\ddagger}$ & $\begin{array}{c}0.051 \\
(0.159)\end{array}$ & $\begin{array}{c}-1.253 * \\
(0.534)\end{array}$ & $\begin{array}{c}-0.233 \\
(0.173)\end{array}$ & $\begin{array}{c}-1.550 * * \\
(0.583)\end{array}$ & $\begin{array}{c}0.159 \\
(0.145)\end{array}$ & $\begin{array}{c}-0.831 \\
(0.490)\end{array}$ \\
\hline Interaction terms & & & & & & \\
\hline Tx satisfaction $\times$ Mod. trust & & $\begin{array}{c}0.233 \\
(0.132)\end{array}$ & & $\begin{array}{c}0.200 \\
(0.145)\end{array}$ & & $\begin{array}{c}0.009 \\
(0.122)\end{array}$ \\
\hline Tx satisfaction $\times$ High trust & & $\begin{array}{c}0.364 * \\
(0.140)\end{array}$ & & $\begin{array}{c}0.366^{*} \\
(0.153)\end{array}$ & & $\begin{array}{r}0.268 * \\
(0.129)\end{array}$ \\
\hline Constant & -1.590 & -2.534 & -0.616 & -1.495 & -1.553 & -1.907 \\
\hline Respondents & 147 & 147 & 147 & 147 & 147 & 147 \\
\hline Observations & 273 & 273 & 273 & 273 & 272 & 273 \\
\hline$R^{2}$ & 0.08 & 0.14 & 0.13 & 0.17 & 0.06 & 0.10 \\
\hline
\end{tabular}

${ }^{\dagger}$ Models present y-standardized coefficients and standard errors in parentheses; ${ }^{\ddagger}$ Mean network trust in doctors is divided into tertiles and the comparison group is low trust; ${ }^{*}=p$ $<0.05 ;{ }^{* *}=p<0.01 ;^{* * *}=p<0.001$ (two-tailed tests).

of Table 4 shows that having larger social networks is significantly associated with higher GAF scores $(b=3.38, p<0.001)$.

The lack of main effects belies significant interactions between treatment experiences and attitudes toward doctors present in the social network. Tables 3 and 4 present regressions that include an interaction term for reporting positive experiences in treatment and network trust in doctors. Every interaction model is significant at $p<0.05$. In the cases of self-esteem, mastery, role functioning, and recovery optimism, high network trust in physicians significantly moderates the effect 
Table 4. Fixed effects linear regression ${ }^{\dagger}$ of recovery optimism and global assessment of functioning (GAF) on the interaction of treatment satisfaction and social network trust in doctors, INMHS.

\begin{tabular}{|c|c|c|c|c|}
\hline & \multicolumn{2}{|c|}{ Recovery optimism } & \multicolumn{2}{|c|}{ GAF } \\
\hline & 1 & 2 & 3 & 4 \\
\hline & $\mathrm{B}(\mathrm{SE})$ & $\mathrm{B}(\mathrm{SE})$ & $\mathrm{B}(\mathrm{SE})$ & $\mathrm{B}(\mathrm{SE})$ \\
\hline Time (years) & $\begin{array}{c}0.095 \\
(0.090)\end{array}$ & $\begin{array}{c}0.102 \\
(0.088)\end{array}$ & $\begin{array}{l}8.818 * * * \\
(1.537)\end{array}$ & $\begin{array}{l}9.051 * * * \\
(1.522)\end{array}$ \\
\hline \multicolumn{5}{|l|}{ Ego characteristics } \\
\hline Education (years) & $\begin{array}{c}0.384 \\
(0.238)\end{array}$ & $\begin{array}{c}0.463 \\
(0.238)\end{array}$ & $\begin{array}{c}2.991 \\
(4.019)\end{array}$ & $\begin{array}{c}4.797 \\
(4.057)\end{array}$ \\
\hline Household income $(\$ 10 \mathrm{~K})$ & $\begin{array}{c}0.090 \\
(0.059)\end{array}$ & $\begin{array}{c}0.085 \\
(0.058)\end{array}$ & $\begin{array}{c}-0.392 \\
(0.989)\end{array}$ & $\begin{array}{c}-0.543 \\
(0.979)\end{array}$ \\
\hline Working fulltime & $\begin{array}{c}0.239 \\
(0.163)\end{array}$ & $\begin{array}{c}0.231 \\
(0.161)\end{array}$ & $\begin{array}{c}6.939 * \\
(2.898)\end{array}$ & $\begin{array}{c}6.373 * \\
(2.878)\end{array}$ \\
\hline Currently married & $\begin{array}{c}-0.078 \\
(0.288)\end{array}$ & $\begin{array}{c}0.031 \\
(0.285)\end{array}$ & $\begin{array}{c}-2.478 \\
(4.855)\end{array}$ & $\begin{array}{c}-1.816 \\
(4.811)\end{array}$ \\
\hline Confidence in doctors & $\begin{array}{c}-0.056 \\
(0.163)\end{array}$ & $\begin{array}{c}-0.082 \\
(0.159)\end{array}$ & $\begin{array}{c}0.568 \\
(2.811)\end{array}$ & $\begin{array}{c}0.319 \\
(2.778)\end{array}$ \\
\hline Receiving treatment & $\begin{array}{c}0.162 \\
(0.159)\end{array}$ & $\begin{array}{c}0.166 \\
(0.157)\end{array}$ & $\begin{array}{c}-2.563 \\
(2.698)\end{array}$ & $\begin{array}{c}-1.722 \\
(2.692)\end{array}$ \\
\hline Treatment satisfaction & $\begin{array}{c}0.137 \\
(0.095)\end{array}$ & $\begin{array}{c}-0.005 \\
(0.117)\end{array}$ & $\begin{array}{c}-0.597 \\
(1.593)\end{array}$ & $\begin{array}{c}-2.933 \\
(1.981)\end{array}$ \\
\hline \multicolumn{5}{|l|}{ Network characteristics } \\
\hline Network size & $\begin{array}{c}-0.085 \\
(0.051)\end{array}$ & $\begin{array}{c}-0.088 \\
(0.050)\end{array}$ & $\begin{array}{l}3.375 * * * \\
(0.858)\end{array}$ & $\begin{array}{l}3.468 * * * \\
(0.850)\end{array}$ \\
\hline Mod. trust in doctors & $\begin{array}{c}-0.208 \\
(0.149)\end{array}$ & $\begin{array}{c}0.001 \\
(0.488)\end{array}$ & $\begin{array}{c}-1.829 \\
(2.490)\end{array}$ & $\begin{array}{r}-16.857 * \\
(8.206)\end{array}$ \\
\hline High trust in doctors ${ }^{\ddagger}$ & $\begin{array}{c}-0.109 \\
(0.158)\end{array}$ & $\begin{array}{r}-1.366 * \\
(0.529)\end{array}$ & $\begin{array}{r}-1.806 \\
(2.711)\end{array}$ & $\begin{array}{r}-14.701 \\
\quad(9.367)\end{array}$ \\
\hline \multicolumn{5}{|l|}{ Interaction terms } \\
\hline Tx satisfaction $\times$ Mod. trust & & $\begin{array}{r}-0.0370 \\
(0.131)\end{array}$ & & $\begin{array}{l}4.385 * \\
(2.209)\end{array}$ \\
\hline Tx satisfaction $\times$ High trust & & $\begin{array}{r}0.338 * \\
(0.139)\end{array}$ & & $\begin{array}{c}3.687 \\
(2.420)\end{array}$ \\
\hline Constant & -3.331 & -3.674 & -58.261 & -73.441 \\
\hline Respondents & 147 & 147 & 145 & 145 \\
\hline Observations & 273 & 273 & 263 & 263 \\
\hline$R^{2}$ & 0.12 & 0.17 & 0.62 & 0.64 \\
\hline
\end{tabular}

${ }^{\dagger}$ Models 1 and 2 present $y$-standardized coefficients, Models 3 and 4 present raw coefficients, and standard errors are in parentheses; ${ }^{\ddagger}$ Mean network trust in doctors is divided into tertiles and the comparison group is low trust; ${ }^{*}=p<0.05 ;{ }^{* *}=p<0.01 ;{ }^{* * *}=p<0.001$ (two-tailed tests).

of treatment experiences. For GAF score, the interaction terms for both moderate and high network trust are large and positive, though only the term for moderate trust achieves significance at $p<0.05$ ( $p$-value for high trust interaction term is $0.13)$.

As shown in Figures 1-5, the effects of having positive treatment experiences on recovery outcomes over time are dependent on the social network culture around 


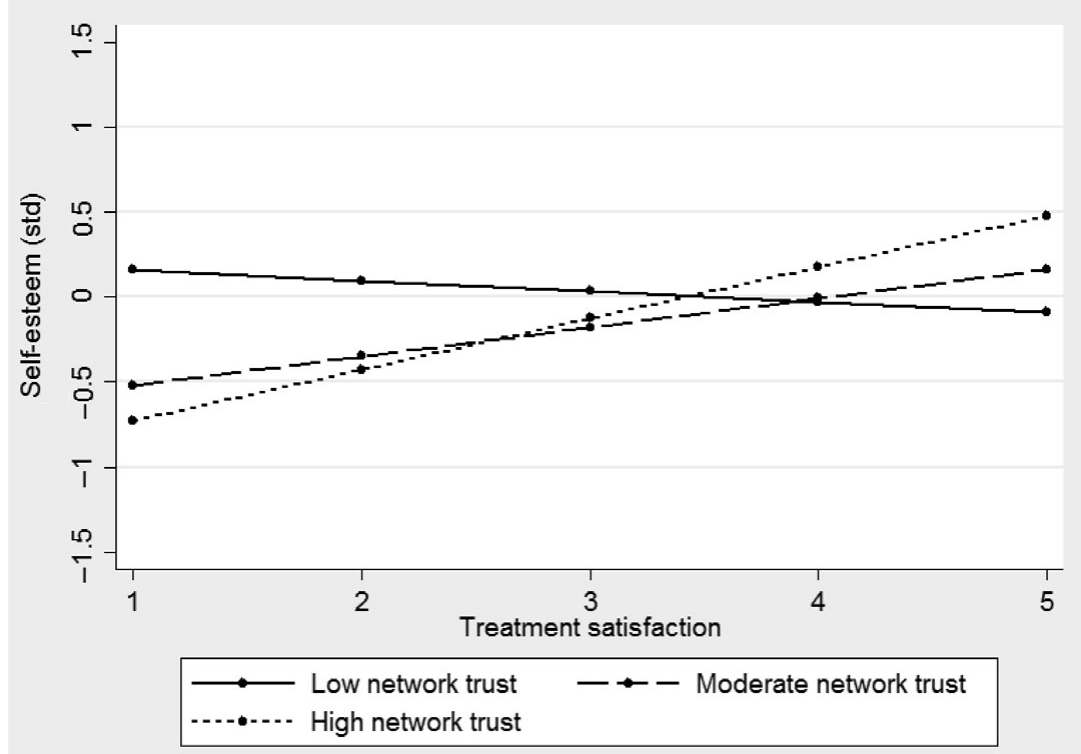

Fig. 1. Moderation of the effect of treatment satisfaction on self-esteem by mean network trust in doctors (based on Model 2 in Table 3), INMHS.

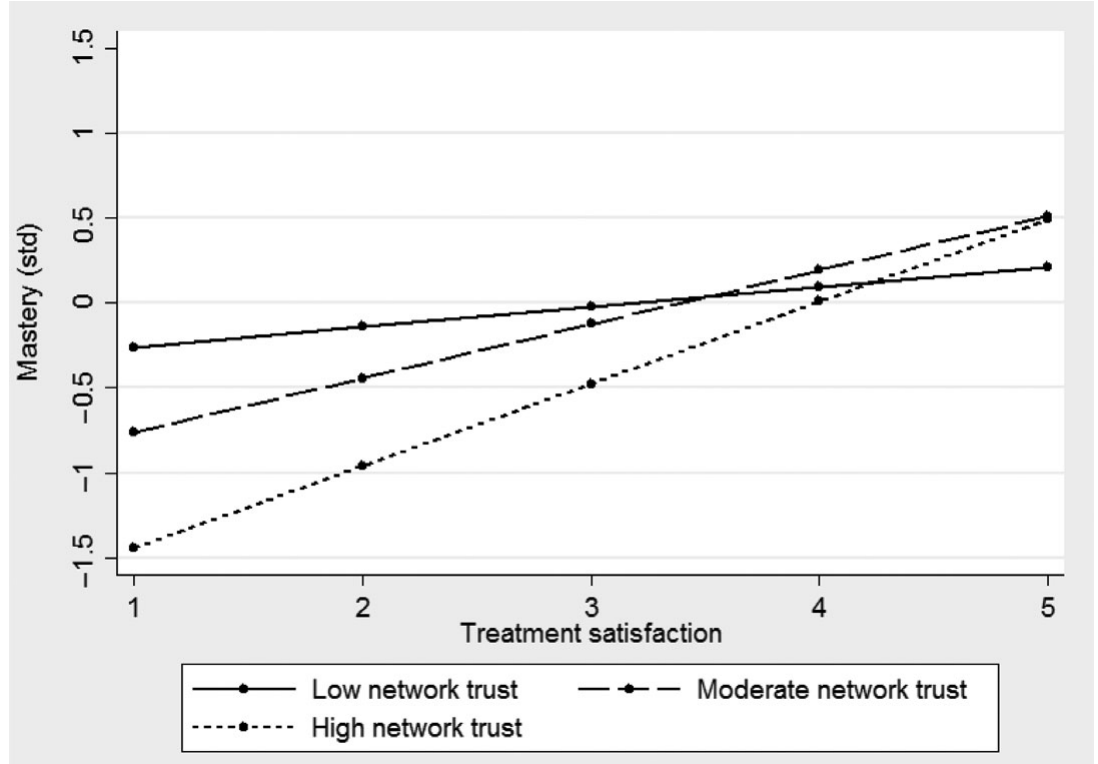

Fig. 2. Moderation of the effect of treatment satisfaction on mastery by mean network trust in doctors (based on Model 4 in Table 3), INMHS. 


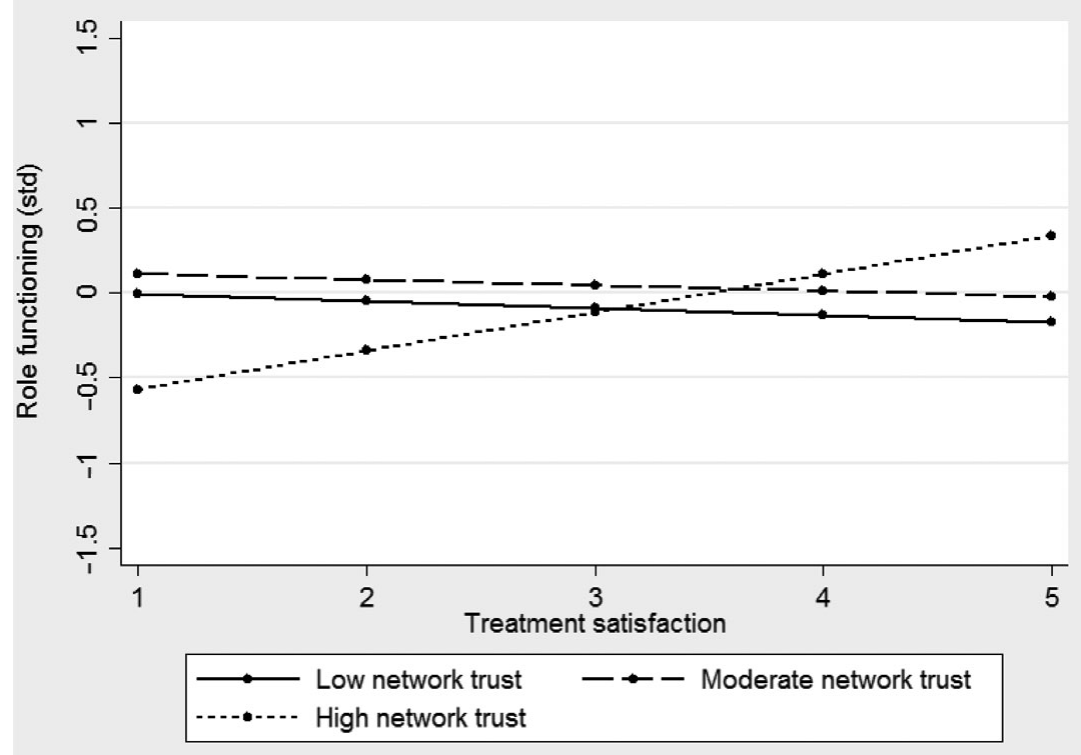

Fig. 3. Moderation of the effect of treatment satisfaction on role functioning by mean network trust in doctors (based on Model 6 in Table 3), INMHS.

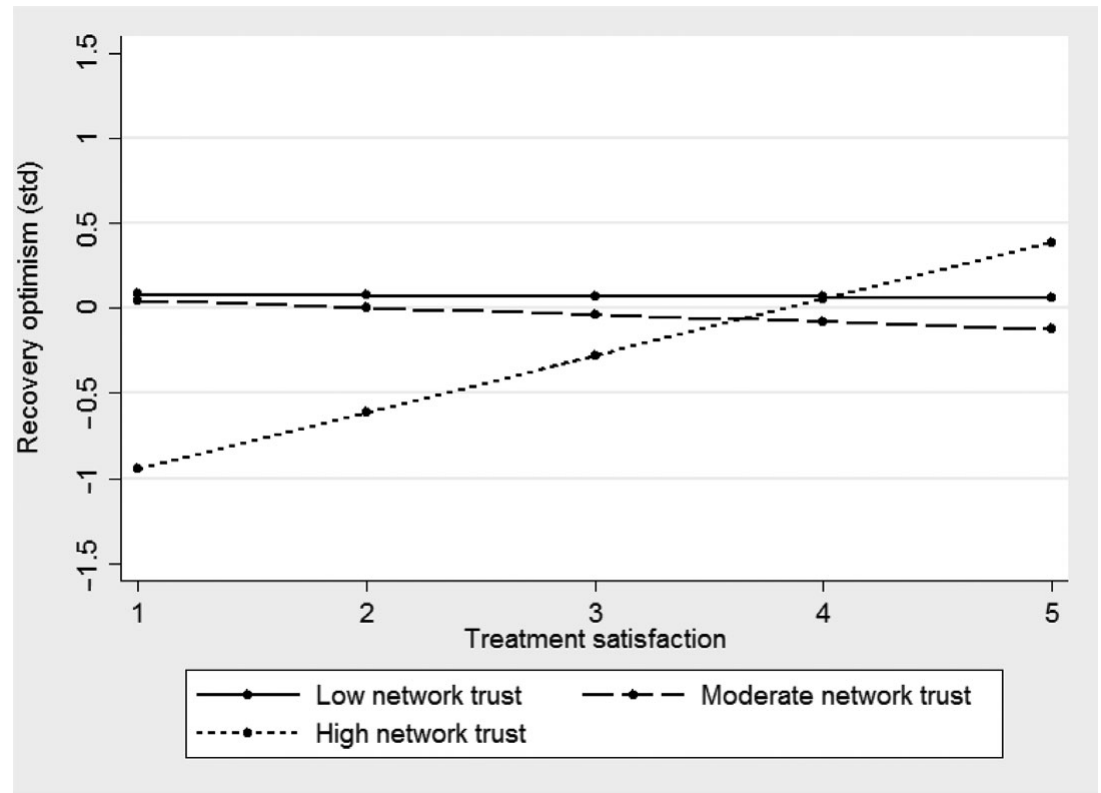

Fig. 4. Moderation of the effect of treatment satisfaction on recovery optimism by mean network trust in doctors (based on Model 2 in Table 4), INMHS. 


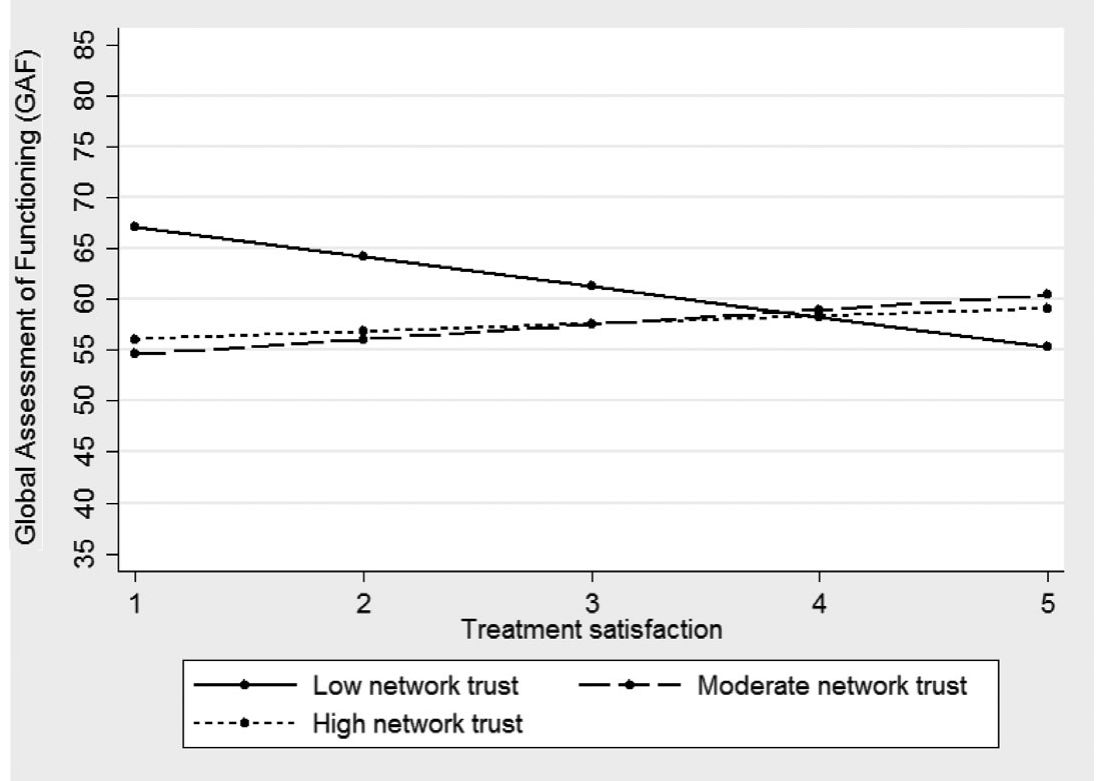

Fig. 5. Moderation of the effect of treatment satisfaction on Global Assessment of Functioning (GAF) by mean network trust in doctors (based on Model 4 in Table 4), INMHS.

confidence in medical institutions. At low levels of network trust in doctors, when alters are skeptical of the value of medical treatment, ego's own experiences in treatment have no impact on psychosocial aspects of recovery over time. However, at moderate and/or high levels of network trust in doctors, more positive treatment experiences are related to better recovery outcomes. Specifically, for individuals whose networks are in the top tertile of network trust in doctors, predicted selfesteem and role functioning increase by about one-quarter of a standard deviation, respectively, for each one-standard deviation increase in treatment satisfaction, net of controls. The relationships between treatment satisfaction and mastery and recovery optimism among people whose networks have high confidence in doctors are even larger in magnitude, with a one-standard deviation increase in positive treatment experiences being associated with a one-half standard deviation increase in these outcomes. Finally, the moderating role of network trust in doctors exhibits a slightly different pattern for GAF. Among individuals with moderate or high network trust in physicians, GAF is predicted to increase slightly over time in conjunction with increases in positive treatment experiences. In contrast, among those whose networks have low confidence in doctors, predicted GAF decreases by about six points for each one-standard deviation increase in positive treatment experiences.

\section{Discussion}

Using data that follows mental health clients for 2 years beyond the point of entry into treatment, we find strong and significant interactions between lay network trust in doctors and treatment experiences in the recovery process. In other words, ties in the community and ties in treatment venues, together, shape outcomes for 
individuals diagnosed with serious mental health problems. Positive relationships and experiences with mental health providers facilitate recovery when the lay network culture is pro-medical, but have no effect (or a negative effect, for one outcome) on the recovery process when the lay network is hostile to medical professionals. In other words, higher satisfaction with treatment is beneficial for recovery outcomes only in the context of a network culture that is supportive of formal medical services. Specifically, the combination of a community-based pro-medical network culture and positive treatment interactions is associated with better self-esteem and mastery, improved role functioning, greater optimism about one's prospects for recovery, and improvements in overall psychological, social, and occupational functioning. In short, the effectiveness of even positive treatment experiences hinges on the moderating influence of cultural characteristics of lay social networks.

Though our research cannot identify the mechanisms underlying the observed interaction between lay network culture and treatment experiences in recovery, there are a number of possible explanations. First, social networks may influence individuals through shared cultural health capital, including attitudes, norms, and values around health and health care utilization. Kadushin $(1962 ; 1966)$ argued that membership in a social network characterized by a positive orientation toward psychotherapy is essential for the efficacy of treatment. He reasoned that psychiatry's professional norms prohibit informal social interaction between practitioners and their clients, in part because an effective therapeutic relationship requires objectivity. However, despite the importance of interactive distance between psychiatrist and client, Kadushin theorizes that cultural distance (i.e. the absence of shared norms, values, and behaviors) and personal distance (i.e. a lack of empathic understanding) threaten the stability of a therapeutic relationship. Therefore, members of lay social networks provide a critical function, transmitting shared culture and experiences that bond mental health practitioners and their clients in the absence of direct informal interaction. More specifically, it is the network of laypeople that socializes individuals to define their problems as psychiatric in nature, recruits them into formal treatment, and supports the goals of therapy. Kadushin (1958) calls this process "bridging the social distance gap (529)," and asserts that cultural and experiential congruence between client and practitioner hinges on access to a community of lay supporters that possesses confidence in psychiatry as a profession, and perhaps in the institution of medicine more broadly.

Second, when a patient's experiences in treatment are incongruous with the attitudes and beliefs of one's network members, this might create cognitive imbalance and distress. Individuals who rely on psychopharmacological treatments, clinical psychotherapy, or other medical interventions to function well in day-to-day life may reframe this reliance as dependence or weakness if they are receiving negative messages from network members about the medical establishment. The result could be reduced self-esteem, mastery, and lower perceptions of one's ability to successfully perform social roles without these medical interventions. Consequently, the antimedical messaging being transmitted through networks might negate some or all of the progress being made toward these elements of recovery in the clinical encounter. In contrast, having a network that is supportive of the medical establishment and confident in doctors could provide validation for an individual's chosen course of action - namely, entry into medical treatment. In other words, having "buy-in" from 
others in the network provides affirmation of the goals and means being pursued in treatment, thereby improving the therapeutic alliance and increasing the efficacy of therapy. Because people with mental illness are already vulnerable and experiencing a major identity shift, they may be particularly susceptible to the psychosocial effects of congruence or incongruence with the predominant culture of their network. Some might even be compelled to rethink their orientation toward formal health care, bringing it in line with their network culture, to maintain cognitive balance and reinforce much-needed supportive relationships (Kenny, 1994; Lazer et al., 2010).

Third, network members' trust in doctors may reveal more about the nature of their own interactions with people with serious mental illness than about the patients' experiences in treatment. Strong confidence in doctors may reflect endorsement of a medical model of illness, and specifically mental illness. This, in turn, could lead to less stigmatization and more sympathetic reactions to disclosure of serious mental illness, and greater willingness to provide emotional or instrumental support (Kadushin, 1958). High levels of trust in the medical establishment may also be indicative of more open and supportive lines of communication about mental illness and related problems between those with more confidence in medical professionals and people recently diagnosed with a psychiatric disorder. It is possible that the efficacy of treatment hinges, in part, on a strong social safety net in the lay community that is supportive of the formal treatment process.

\section{Limitations and future directions}

A limitation of this study is the age of the data, which were collected prior to the Patient Protection and Affordable Care Act and many important changes in the mental health treatment system. Increasingly, the functions of the public mental health system have been taken over by the criminal justice system, blurring the lines between psychiatric treatment and criminalization (Fisher et al., 2016). Many individuals with serious mental illness now receive treatment in jails and prisons. At the same time, continuing deinstitutionalization and shrinking public funding for mental health initiatives (especially for the most ill individuals) have reduced access to care in the community, increasing family responsibility for people with serious mental illness (Scheid, 2016; Schutt, 2016). It is not certain how these changes might have affected the data and therefore the results of this paper, but it is clear that lay social networks - and especially family members - have become an increasingly important and influential factor in the contemporary landscape of mental health treatment and recovery. In the future, the moderating role of lay network culture on the efficacy of positive treatment interactions should be examined using larger and newer datasets.

In addition, we operationalize membership in a network culture that is supportive of mental health treatment using a single limited measure - trust in doctors. Ideally, we would employ a more multidimensional strategy for assessing cultural orientation toward medical and mental health treatment. This might include measurement of alters' level of education, knowledge of psychiatric disorders, experience using mental health services, the degree to which they hold stigmatizing attitudes about people with mental illness, and a scale capturing different dimensions of attitudes toward medical professionals. Though the egocentric social network design has 
many advantages (e.g. ability to collect specific information about regular interaction partners across a variety of social contexts), its high respondent burden prohibits the inclusion of a large number of name interpreters (i.e. items asked about each alter). Nonetheless, a study that was targeted toward measurement of cultural health capital, in conjunction with social network methods, could better capture network culture around health and medicine.

Finally, it is well known that there are systematic racial and ethnic inequalities in experiences in the health care system and distinct attitudes toward doctors and the medical establishment (Boulware et al., 2003; Wright \& Perry, 2010). Because the majority of the INMHS sample was White, it was not possible to test for group differences in the effects of patient or network trust in doctors on recovery outcomes, or to determine whether network culture and treatment experiences interact in unique ways for different racial and ethnic groups. These research questions should be addressed in the future in a way that is sensitive to racial and ethnic health and health care disparities and experiences at the intersection of different race, gender, and socioeconomic statuses.

\section{Conclusion}

Despite these limitations, the INMHS is unique in its longitudinal design, beginning with first major entry into treatment - and its detailed assessment of characteristics of network ties across a variety of community and treatment contexts. Moreover, this research makes important contributions to our understanding of how lay and professional contexts interact during the early stages of an episode of illness. Our findings support Kadushin's (1966) classic theory of the influence of personal social networks in pathways to formal care. Rather than seeing the decision to enter mental health services as an individual, rational choice, he saw membership in networks with "cultural and psychiatric sophistication (796)" as a critical determinant of the definition of and response to psychiatric symptoms (see also Pescosolido, 1992). Here, we extend this theory to more advanced stages of the illness career, demonstrating that the effects of treatment interactions up to 2 years after entry into care are moderated by the presence of lay networks that are supportive or skeptical of medical professionals. Moreover, our findings illustrate the long reach of lay network culture into even the most private and formalized relationship - that of provider and patient.

From a practical standpoint, then, it may be necessary to cultivate lay supporters of psychiatry among clients without naturally occurring mental health allies. Along these lines, participation in group therapy and programs like the club house model may be beneficial for creating an artificial community that is knowledgeable about mental health services and supportive of clients' therapeutic goals and recovery (Jackson, 2001; Staples \& Stein, 2008). Alternatively, there are a number of evidencebased models that are aimed at increasing support for patients' recovery goals among existing networks, including community support systems approaches, consumercentered family models, and related evidenced-based interventions for families (e.g. psychoeducational therapy). These more holistic and inclusive treatment models embrace the critical role of lay supporters and community integration in treatment efficacy (Lucksted et al., 2012). For example, participation in NAVIGATE - an 
innovative, comprehensive community care program that includes psychoeducation for families - was recently found to be associated with better recovery outcomes (i.e. treatment retention, improvement in quality of life and symptomatology, improvement in vocational and educational functioning) compared to usual community care (Kane et al., 2016). These kinds of evidence-based interventions are designed to facilitate collaborative management of people with serious mental illness by professionals and family members, including coordinating treatment, ensuring medication compliance, and involving family members in treatment planning (Dixon et al., 2014; Lucksted et al., 2012). Our findings underscore that achieving buy-in from members of lay community networks is critical for the success of therapeutic interventions.

\section{Acknowledgments}

We acknowledge financial support from the National Institute of Mental Health (grants K01MH00849, R29MH44780, and R24MH51669), the Indiana Consortium for Mental Health Services Research, and the Indiana University Network Science Institute. We thank Mary Hannah for her valuable assistance. Direct correspondence to the first author, Department of Sociology, Ballantine Hall 744, 1020 E. Kirkwood Ave., Bloomington, IN 47405. E-mail: blperry@indiana.edu.

\section{References}

Abbott, K. M., Bettger, J. P., Hanlon, A., \& Hirshman, K. B. (2012). Factors associated with health discussion network size and composition among elderly recipients of long-term services and supports. Health Communication, 27(8), 784-793.

Adair, C. E., McDougall, G. M., Mitton, C. R., Joyce, A. S., Wild, C., Gordon, A., ... Beckie, A. (2005). Continuity of care and health outcomes among persons with severe mental illness. Psychiatric Services, 56(9), 1061-1069.

Adams, J. R., Drake, R. E., \& Wolford, G. L. (2007). Shared decision-making preferences of people with severe mental illness. Psychiatric Services, 58(9), 1219-1221.

Ajrouch, K. J., Antonucci, T. C., \& Janevic, M. R. (2001). Social networks among blacks and whites: The interaction between race and age. Journal of Gerontology: Social Sciences, 56(2), 112-118.

Allen, M. L., Lê Cook, B., Carson, N., Interian, A., La Roche, M., \& Alegría, M. (2015). Patientprovider therapeutic alliance contributes to patient activation in community mental health clinics. Administration and Policy in Mental Health and Mental Health Services Research, Epub 2015 May 12. doi: 10.1007/s10488-015-0655-8.

Allison, P. D. (2005). Fixed effects regression methods for longitudinal data using SAS. Cary, NC: SAS Institute.

Barrowclough, C., Meier, P., Beardmore, R., \& Emsley, R. (2010). Predicting therapeutic alliance in clients with psychosis and substance misuse. Journal of Nervous \& Mental Disease, 198(5), 373-377.

Boulware, L. E., Cooper, L. A., Ratner, L. E., LaVeist, T. A., \& Powe, N. R. (2003). Race and trust in the health care system. Public Health Reports, 118(4), 358.

Bourdieu, P. (1977). Outline of a theory of practice. Cambridge: Cambridge University Press.

Bourdieu, P. (1986). The forms of capital. In J. G. Richardson (Ed.), Handbook of theory and research for the sociology of education (pp. 81-93). New York: Greenwood.

Bourdieu, P. (1990). The logic of practice. Stanford, California: Stanford University Press 
Bracke, P., Christiaens, W., \& Verhaeghe, M. (2008). Self-esteem, self-efficacy, and the balance of peer support among persons with chronic mental health problems. Journal of Applied Social Psychology, 38(2), 436-459.

Carpentier, N., \& Bernard, P. (2011). The complexities of help-seeking: Exploring challenges through a social network perspective. In B. A. Pescosolido, J. K. Martin, J. D. McLeod, \& A. Robgers (Eds.), The handbook of the sociology of health, illness, and healing (pp. 465-479). New York: Springer.

Carpentier, N., Lesage, A., \& White, D. (1999). Family influence on the first stages of the trajectory of patients diagnosed with severe psychiatric disorders. Family Relations, 48(4), 397-403.

Centola, D. (2010). The spread of behavior in an online social network experiment. Science, 329(5996), 1194-1197.

Clark, T. S., \& Linzer, D. A. (2015). Should I use fixed or random effects? Political Science Research and Methods, 3(02), 399-408.

Cruz, M., \& Pincus, H. A. (2002). Research on the influence that communication in psychiatric encounters has on treatment. Psychiatric Services, 53(10), 1253-1265.

Cummings, S. M., \& Kropf, N. P. (2009). Formal and informal support for older adults with severe mental illness. Aging \& Mental Health, 13(4), 619-627.

Curtis, L. C., Wells, S. M., Penney, D. J., Ghose, S. S., Mistler, L. A., Mahone, I. H., . . Lesko, S. (2010). Pushing the envelope: Shared decision making in mental health. Psychiatric Rehabilitation Journal, 34(1), 14-22.

Cusack, J., Deane, F. P., Wilson, C. J., \& Ciarrochi, J. (2004). Who influences men to go to therapy? Reports from men attending psychological services. International Journal for the Advancement of Counselling, 26(3), 271-283.

Davidson, L., O’Connell, M. J., Tondora, J., Lawless, M., \& Evans, A. C. (2005). Recovery in serious mental illness: A new wine or just a new bottle? Professional Psychology: Research and Practice, 36(5), 480.

Dixon, L., McFarlane, W. R., Lefley, H., Lucksted, A., Cohen, M., Falloon, I., ... Sondheimer, D. (2014). Evidence-based practices for services to families of people with psychiatric disabilities. Psychiatric Services, 52(7), 903-910.

Dracup, K. (2013). Cardiac rehabilitation: The role of social support in recovery and compliance. In S. A. Schumaker \& S. M. Czajkowski (Eds.), Social support and cardiovascular disease (pp. 333-354). New York, NY: Springer Science \& Business Media.

Duncan, G. J., Wilkerson, B., \& England, P. (2006). Cleaning up their act: The effects of marriage and cohabitation on licit and illicit drug use. Demography, 43(4), 691-710.

Fisher, W. H., Geller, J. L., \& McMannus, D. L. (2016). Same problem, different century: Issues in recreating the functions of public psychiatric hospitals in community-based settings. In B. L. Perry (Ed.). 50 years after deinstitutionalization: Mental illness in contemporary communities (pp. 3-25). Bingley: Emerald Group Publishing Limited.

Franks, M. M., Stephens, M. A., Rook, K. S., Franklin, B. A., Keteyian, S. J., \& Artinian, N. T. (2006). Spouses' provision of health-related support and control to patients participating in cardiac rehabilitation. Journal of Family Psychology, 20(2), 311-318.

Gant, J. R., Calsyn, R. J. \& Winter, J. (1999). Predicting agreement between providers and recipients of social support. Journal of Social Behavior and Personality, 14(4), 523.

Hansen, M. C., \& Aranda, M. P. (2012). Sociocultural influences on mental health service use by Latino older adults for emotional distress: Exploring the mediating and moderating role of informal social support. Social Science \& Medicine, 75(12), 2134-2142.

Heider, F. (1958). The psychology of interpersonal relations. New York: John Wiley.

Hendryx, M., Green, C. A., \& Perrin, N. A. (2009). Social support, activities, and recovery from serious mental illness: STARS study findings. Journal of Behavioral Health Services Research, 36(3), 320-329. 
Horvath, A. O., Del Re, A. C., Flückiger, C., \& Symonds, D. (2011). Alliance in individual psychotherapy. Psychotherapy, 48(1), 9-16.

Hummon, N. P., \& Doreian, P. (2003). Some dynamics of social balance processes: Bringing heider back into balance theory. Social Networks, 25(1), 17-49.

Hurlbert, J. S., Haines, V. S., \& Beggs, J. J. (2000). Core networks and tie activation: What kinds of routine networks allocate resources in nonroutine situations? American Sociological Review, 65(4), 598-618.

Jackson, R. L. (2001). The clubhouse model: Empowering applications of theory to generalist practice. Boston: Brooks/Cole Publishing Company.

Johansson, H., \& Eklund, M. (2006). Helping alliance and early dropout from psychiatric outpatient care: The influence of patient factors. Social Psychiatry and Psychiatric Epidemiology, 41(2), 140-147.

Kadushin, C. (1958). Individual decisions to undertake psychotherapy. Administrative Science Quarterly, 3(3), 379-411.

Kadushin, C. (1962). Social distance between client and professional. American Journal of Sociology, 67(5), 517-531.

Kadushin, C. (1966). The friends and supporters of psychotherapy: On social circles in urban life. American Sociological Review, 31(6), 786-802.

Kane, J. M., Robinson, D. G., Schooler, N. R., Mueser, K. T., Penn, D. L., Rosenheck, R. A., ... Marcy, P. (2016). Comprehensive versus usual community care for first-episode psychosis: 2-year outcomes from the NIMH RAISE early treatment program. American Journal of Psychiatry, 173(4), 362-372.

Kenny, C. B. (1994). The microenvironment of attitude change. The Journal of Politics, 56(3), 715-728.

Kreyenbuhl, J., Nossel, I. R., \& Dixon, L. B. (2009). Disengagement from mental health treatment among individuals with schizophrenia and strategies for facilitating connections to care: A review of the literature. Schizophrenia Bulletin, 35(4), 696-703.

Laugharne, R., \& Priebe, S. (2006). Trust, choice, and power in mental health: A literature review. Psychiatry and Psychiatric Epidemiology, 41(11), 846-852.

Lazer, D., Rubineau, B., Chetkovich, C., Katz, N., \& Neblo, M. (2010). The coevolution of networks and political attitudes. Political Communication, 27(3), 248-274.

Lewis, M. A., \& Butterfield, R. M. (2005). Antecedents and reactions to health-related social control. Personality and Social Psychology Bulletin, 31(3), 416-427.

Lewis, M. A., \& Butterfield, R. M. (2007). Social control in marital relationships: Effect of one's partner on health behaviors. Journal of Applied Social Psychology, 37(2), 298319.

Lindsey, M. A., Barksdale, C. L., Lambert, S. F., \& Ialongo, N. S. (2010). Social network influences on service use among urban, African American youth with mental health problems. Journal of Adolescent Health, 47(4), 367-373.

Lively, K. J. \& Smith, C. L. (2011). Identity and illness. In B. Pescosolido, J. K. Martin, J. D. McLeod, \& A. Rogers (Eds.), Handbook of the sociology of health, illness, and healing (pp. 505-525). New York: Springer.

Lo, M.-c. M., \& Stacey, C. L. (2008). Beyond cultural competency: Bourdieu, patients, and clinical encounters. Sociology of Health and Illness, 30(5), 741-755.

Lucksted, A., McFarlane, W., Downing, D., \& Dixon, L. (2012). Recent developments in family psychoeducation as an evidence-based practice. Journal of Marital and Family Therapy, 38(1), 101-121.

Markowitz, F. E. (2001). Modeling processes in recovery from mental illness: Relationships between symptoms, life satisfaction, and self-concept. Journal of Health and Social Behavior, 42(1), 64-79. 
Marsden, P. (1987). Core discussion networks of Americans. American Sociological Review, 52(1), 122-131.

McCabe, R., \& Priebe, S. (2004). The therapeutic relationship in the treatment of severe mental illness: A review of methods and findings. International Journal of Social Psychiatry, 50(2), 115-128.

Mezzina, R., Davidson, L., Borg, M., Marin, I., Topor, A., \& Sells, D. (2006). The social nature of recovery: Discussion and implications for practice. American Journal of Psychiatric Rehabilitation, 9(1), 63-80.

National Survey on Drug Use and Health (NSDUH). (2009). Results from the 2008 national survey on drug use and health: National findings. Substance Abuse and Mental Health Services Administration, U.S. Department of Health and Human Services. Retrieved from http://goo.gl/ZIUJqa.

National Survey on Drug Use and Health (NSDUH). (2014). Behavioral health trends in the United States: Results from the 2014 national survey on drug use and health. Substance Abuse and Mental Health Services Administration, U.S. Department of Health and Human Services. Retrieved from http://www.samhsa.gov/data/sites/default/files/NSDUH-FRR12014/NSDUH-FRR1-2014.pdf.

Padgett, D. K., Henwood, B., Abrams, C., \& Drake, R. E. (2008). Social relationships among persons who have experienced serious mental illness, substance abuse, and homelessness: Implications for recovery. American Journal of Orthopsychiatry, 78(3), 333-339.

Pearlin, L. I., \& Schooler, C. (1978). The structure of coping. Journal of Health and Social Behavior, 19(1), 2-21.

Peek, M. K., \& O’Neill, G. S. (2001). Networks in later life: An examination of race differences in social support networks. International Journal of Aging and Human Development, 52(3), 197-219.

Pernice-Duca, F. (2010). Family network support and mental health recovery. Journal of Marital \& Family Therapy, 36(1), 13-27.

Perry, B. L., \& Pescosolido, B. A. (2010). Functional specificity in discussion networks: The influence of general and problem-specific networks on health outcomes. Social Networks, 32(4), 345-357.

Perry, B. L., \& Pescosolido, B. A. (2012). Social network dynamics and biographical disruption: The case of "first-timers" with mental illness. American Journal of Sociology, 118(1), 134175.

Perry, B. L., \& Pescosolido, B. A. (2015). Social network activation: The role of health discussion partners in recovery from mental illness. Social Science \& Medicine, 125, 116128.

Perry, B. L., Pullen, E., \& Pescosolido, B. A. (2016). At the intersection of lay and professional social networks: How community ties shape perceptions of mental health treatment providers. Global Mental Health Journal, 3, 1-17.

Pescosolido, B. A. (1992). Beyond rational choice: The social dynamics of how people seek help. American Journal of Sociology, 97, 1096-1138.

Pescosolido, B. A. (1996). Bringing the 'community' into utilization models: How social networks link individuals to changing systems of care. In J. J. Kronenfeld (Ed.), Research in the sociology of health care, Vol. 13 (pp. 171-198). Greenwich: JAI Press.

Pescosolido, B. A. (2006). Of pride and prejudice: the role of sociology and social networks in integrating the health sciences. Journal of Health and Social Behavior, 47(3), 189-208.

Pescosolido, B. A., Gardner, C. B., \& Lubell, K. M. (1998a). How people get into mental health services: Stories of choice, coercion and "muddling through" from "first-timers". Social Science \& Medicine, 46(2), 275-286.

Pescosolido, B. A., Wright, E. R., Alegria, M., \& Vera, M. (1998b). Social networks and patterns of use among the poor with mental health problems in Puerto Rico. Medical Care, 36(7), 1057-1072. 
Pullen, E., Perry, B., \& Oser, C. (2014). African American women's preventative care usage: The role of social support and racial experiences and attitudes. Sociology of Health \& Illness, 36(7), 1037-1053.

Roe, D., Goldblatt, H., Baloush-Klienman, V., Swarbrick, M., \& Davidson, L. (2009). Why and how people decide to stop taking prescribed psychiatric medication: Exploring the subjective process of choice. Psychiatric Rehabilitation Journal, 33(1), 38-46.

Rogers, C. R. (1951). Client-centered therapy. Cambridge: Riverside Press.

Rosenberg, M. (1965). Society and the adolescent self-image. Princeton: Princeton University Press.

Scheid, T. L. (2016). An institutional analysis of public sector mental health in the post-deinstitutionalization era. In B. L. Perry (Ed.). 50 years after deinstitutionalization: Mental illness in contemporary communities (pp. 63-87). Bingley: Emerald Group Publishing Limited.

Schutt, R. K. (2016). Social environment and mental illness: The progress and paradox of deinstitutionalization. In B. L. Perry (Ed.). 50 years after Deinstitutionalization: Mental illness in contemporary communities (pp. 91-118). Bingley: Emerald Group Publishing Limited.

Shim, J. K. (2010). Cultural health capital a theoretical approach to understanding health care interactions and the dynamics of unequal treatment. Journal of Health and Social Behavior, 51(1), 1-15.

Spitzer, R. L., Williams, J. B. W., Gibbon, M., \& First, M. B. (1990). Structured clinical interview for DSM-III-R, patient edition/non-patient edition (SCID-P/SCID-NP). Washington DC: American Psychiatric Press, Inc.

Spoont, M. R., Nelson, D. B., Murdoch, M., Rector, T., Sayer, N. A., Nugent, S., \& Westermeyer, J. (2014). Impact of treatment beliefs and social network encouragement on initiation of care by VA service users with PTSD. Psychiatric Services, 65(5), 654-662.

Staples, L., \& Stein, R. (2008). The clubhouse model: Mental health consumer-provider partnerships for recovery. Advances in Medical Sociology: Patients, Consumers and Civil Society, 10, 177-196.

Startup, M., Jackson, M. C., \& Bendix, S. (2002). The concurrent validity of the global assessment of functioning. British Journal of Clinical Psychology, 41(4), 417-422.

Thoits, P. A. (2011). Perceived social support and the voluntary, mixed, or pressured use of mental health services. Society and Mental Health, 1(1), 4-19.

Trotter, R. T., Baldwin, J. A., \& Bowen, A. M. (1995). Network structure and proxy network measures of HIV, drug and incarceration risks for active drug users. Connections, 18(1), 88-103.

Uchino, B. N. (2004). Social support and physical health: Understanding the health consequences of relationship. New Have, CT: Yale University Press.

Umberson, D. (1992). Gender, marital status and the social control of health behavior. Social Science and Medicine, 34(8), 907-917.

Villatoro, A. P., \& Aneshensel, C. S. (2014). Family influences on the use of mental health services among African Americans. Journal of Health and Social Behavior, 55(2), 161-180.

Wild, T. C., Cunningham, J. A., \& Ryan, R. M. (2006). Social pressure, coercion, and client engagement at treatment entry: A self-determination theory perspective. Addictive Behaviors, 31(10), 1858-1872.

Wright, E. R., \& Perry, B. L. (2010). Medical sociology and health services research past accomplishments and future policy challenges. Journal of Health and Social Behavior, 51(Suppl. 1), S107-S119. 